This is the peer reviewed version of the following article: Angew. Chem. 2009,121, 821-824 which has been published in final form at: https://doi.org/10.1002/ange.200803736.

This article may be used for non-commercial purposes in accordance with Wiley Terms and Conditions for Use of Self-Archived Versions.

Niedervalente

DOI: 10.1002/anie.200((will be filled in by the editorial staff))

Lewis-Säure

\title{
Erste Reaktion von Dizinkocen unter Erhalt der Zn-Zn-Bindung
}

\author{
Daniella Schuchmann, Ulrich Westphal, Stephan Schulz, * Ulrich Flörke, Dieter Bläser und Roland \\ Boese
}

((Prof. Dr. E. Niecke zum 70. Geburtstag gewidmet))

Seit der bahnbrechenden Synthese von $\mathrm{Cp}^{*}{ }_{2} \mathrm{Zn}_{2} \mathbf{1}^{[1]}$ der ersten Verbindung mit einer Zn-Zn-Bindung, durch Carmona et al. im Jahr 2004, wurden vier weitere Verbindungen des Typs $\mathrm{R}_{2} \mathrm{Zn}_{2}$ durch Verwendung sterisch anspruchsvoller Substituenten $(\mathrm{R}=\mathrm{Et}-$ $\mathrm{Me}_{4} \mathrm{Cp},{ }^{[2]} \quad\left[2,6-\left(2,6-i-\mathrm{Pr}_{2} \mathrm{C}_{6} \mathrm{H}_{3}\right)-\mathrm{C}_{6} \mathrm{H}_{3}\right],{ }^{[3]} \quad[\{(2,6-i-$ $\left.\left.\left.\mathrm{Pr}_{2} \mathrm{C}_{6} \mathrm{H}_{3}\right) \mathrm{N}(\mathrm{Me}) \mathrm{C}\right\}{ }_{2} \mathrm{CH}\right],{ }^{[4]}\left[\left(2,6-i-\mathrm{Pr}_{2} \mathrm{C}_{6} \mathrm{H}_{3}\right) \mathrm{N}(\mathrm{Me}) \mathrm{C}\right],{ }^{[5]}\{\mathrm{Me} 2 \mathrm{Si}[\mathrm{N}-$ $\left.\left.\left(2,6-i-\mathrm{Pr}_{2} \mathrm{C}_{6} \mathrm{H}_{3}\right)\right]_{2}\right\},{ }^{[6]} \quad$ 1,2-Bis[(2,6-diisopropylphenyl)imino]acenaphthen (dpp-bian ${ }^{[7]}$ ) synthetisiert und strukturell charakterisiert. Obwohl die Natur der Zn-Zn-Bindung insbesondere von $\mathrm{Cp}$ substituierten Verbindungen von verschieden Arbeitsgruppen untersucht wurde, ${ }^{[8]}$ ist über die Reaktivität derartiger Verbindungen nur wenig bekannt. Lediglich Carmona et al. berichtete über Reaktionen von 1 mit $\mathrm{H}_{2} \mathrm{O}, t$-BuOH, $\mathrm{NCXyl}{ }^{[1]}$ und anderen Lewis-Basen $\left(\mathrm{NMe}_{3}\right.$, Pyridin, $\left.\mathrm{PMe}_{3}, \ldots\right),{ }^{[2]}$ bei denen entweder keine Reaktion oder aber Disproportionierung in elementares Zink und der entsprechenden $\mathrm{Zn}(\mathrm{II})$-Verbindung beobachtet wurde. Reaktionen mit $\mathrm{ZnR}_{2}(\mathrm{R}=\mathrm{Me}$, Mes) führen $\mathrm{zu}$ Halbsandwichkomplexen $\mathrm{Cp} * \mathrm{ZnR}$ und mit Jod erfolgt Oxidation unter Bildung von $\mathrm{Cp}^{*}{ }_{2} \mathrm{Zn}$ und $\mathrm{ZnI}_{2}$, während bei Umsetzungen mit $\mathrm{H}_{2}, \mathrm{CO}, \mathrm{CO}_{2}$ keine Reaktion beobachtet wurde. Diesen Untersuchungen zufolge erscheinen Reaktionen unter Erhalt der Zn-Zn-Bindung problematisch.

Wir interessieren uns seit kurzem für Organozinkverbindungen mit sterisch weniger anspruchsvoll substituierten, N,N'chelatisierenden Amidinatliganden, ${ }^{[9]}$ aus denen u. a. Verbindungen mit $\mathrm{Zn}-\mathrm{Zn}$-Bindung synthetisiert werden sollen. Parallel dazu untersuchen wir die Reaktivität der Zn-Zn-Bindung. Hierbei richtete sich unser Fokus auf Dizinkocen $\mathrm{Cp}^{*}{ }_{2} \mathrm{Zn}_{2}$ 1, da diese Verbindung aufgrund der sterischen und elektronischen Flexibilität des $\mathrm{Cp}^{*}$ Liganden am aussichtsreichsten für Folgereaktionen erscheint. ${ }^{[10]} \mathrm{Da}$ wir in den vergangenen Jahren detailliert die Lewis-Azidität von Gruppe 13-Verbindungen untersuchten, interessierten wir uns zu-

[*] Prof. Dr. S. Schulz. D. Schuchmann, Dr. U. Westphal, D. Bläser, Prof. R. Boese

Institut für Anorganische Chemie,Universität DuisburgEssen

Universitätsstr. 5-7, S07 S03 C30, 45117 Essen

Fax: (+) 201-183 3830

E-mail: stephan.schulz@uni-due.de

Homepage: http://www.uni-duisburg-

essen.de/chemie/institute.shtml

\section{Dr. U. Flörke}

Department Chemie, Universität Paderborn

Warburger Str. 100, J6.214, 33098 Paderborn

[**] S. Schulz dankt der DFG für die finanzielle Förderung, D. Schuchmann dem Fonds der Chemischen Industrie für ein Doktorandenstipendium.

Supporting information for this article is available on the WWW under http://www.angewandte.org or from the author. nächst für die Lewis-Azidität von $\mathrm{Cp}^{*}{ }_{2} \mathrm{Zn}_{2}$ 1. Hierzu untersuchten wir dessen Reaktion mit der starken Lewis-Base 4Dimethylaminopyridin (dmap), mit der wir in früheren Untersuchungen zahlreiche Addukte synthetisieren konnten. ${ }^{[11]}$ Wir berichten hier über die Bildung des unerwarteten Lewis Säure-Base Adduktes 2, in dem zwei dmap-Moleküle unter Erhalt der Zn-ZnBindung geminal an ein Zn-Zentrum binden. Zum Vergleich wurde die analoge Reaktion mit $\mathrm{Cp}^{*}{ }_{2} \mathrm{Zn}$, die unter Bildung von $\mathrm{Cp}{ }_{2} \mathrm{Zn}$ (dmap) 3 verläuft, untersucht.

1 reagiert sowohl mit zwei Äquivalenten als auch mit einem Überschuß dmap bei $0{ }^{\circ} \mathrm{C}$ in einer Lösung aus Toluol und Pentan (1:4) zu 2, welches nach Kristallisation bei $-30{ }^{\circ} \mathrm{C}$ in Form hellgelber Kristalle isoliert wurde. Im Unterschied zu den geschilderten Reaktionen mit anderen Lewis-Basen erfolgt keine Disproportionierung der niedervalenten $Z n$-Atome. ${ }^{[2]}$ Die analoge Reaktion von $\mathrm{Cp}_{2} \mathrm{Zn}$ mit dmap ergibt Cp* ${ }_{2} \mathrm{Zn}$-dmap 3.

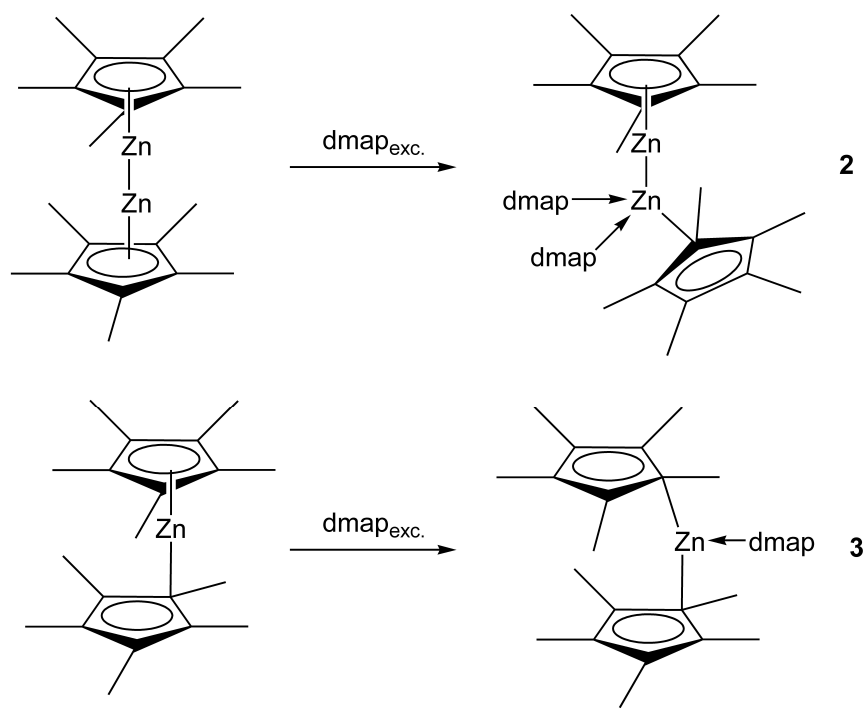

Scheme 1. Synthese der dmap-Addukte $C p^{*} \mathrm{Zn}-\mathrm{Zn}(\mathrm{dmap})_{2} \mathrm{Cp}^{*} 2$ und $C p^{*}$ Zn-dmap 3

${ }^{1} \mathrm{H}$ - und ${ }^{13} \mathrm{C}-\mathrm{NMR}$ Spektren von $\mathbf{2}$ und $\mathbf{3}$ zeigen Resonanzen der Cp*-Substituenten sowie von dmap. Das Vorliegen einer hydridverbrückten Spezies für 2 kann ausgeschlossen werden, da weder das ${ }^{1} \mathrm{H}$ NMR Spektrum eine Resonanz im Bereich zwischen 4 und 5 ppm noch das IR Spektrum eine für Zinkhydride typische Bande im Bereich zwischen 1650 und $1900 \mathrm{~cm}^{-1}$ zeigt. ${ }^{[12]}$ Einkristalle von 2, die aus einer Lösung in Pentan/Toluol erhalten wurden, zeigen im ${ }^{1} \mathrm{H}$ NMR Spektrum zusätzliche Resonanzen für Toluol. Für die $\mathrm{Cp}^{*}$ Gruppen wird bei $25{ }^{\circ} \mathrm{C}$ jeweils nur ein Signal detektiert ( $\delta 2.03$ 2, $2.08 \mathrm{ppm}$ 3), was auf schnelle Austauschprozesse zwischen den chemisch nicht äquivalenten $\mathrm{Cp}^{*}$-Liganden in 2 sowie auf $\eta^{5}$ - 
gebundene $\mathrm{Cp}^{*}$-Gruppen bzw. auf eine fluktuierende Struktur der $\mathrm{Cp}{ }^{*}$-Gruppen hindeutet. Erst bei $-95{ }^{\circ} \mathrm{C}$ spaltet die $\mathrm{Cp}^{*}$-Resonanz von 2 in zwei einzelne breite Resonanzen $(\delta 2.48,2.73)$ gleicher Intensität auf. Zudem zeigen temperaturabhängigen ${ }^{1} \mathrm{H}-\mathrm{NMR}$ Spektren von 2 eine starke Abhängigkeit der chemischen Verschiebung der $\mathrm{Cp}^{*}$ - und dmap-Protonenresonanzen von der Messtemperatur, was auf temperaturabhängige Gleichgewichtsreaktionen hindeutet. ${ }^{[13]}$ Aus diesen ${ }^{1} \mathrm{H}-\mathrm{NMR}$ Spektren wurde die Dissoziationsenthalpie von $2\left(\Delta \mathrm{H}_{\text {Diss }}=-40.4 \mathrm{~kJ} / \mathrm{mol}\right)$ in Lösung berechnet. ${ }^{[14]}$ Die Dissoziationsenthalpie von $3\left(\Delta \mathrm{H}_{\text {Diss }}=-50.6 \mathrm{~kJ} / \mathrm{mol}\right)$ wurde auf analoge Weise experimentell bestimmt. Sie belegt die vergleichsweise größere Lewis-Basizität von $\mathrm{Cp}_{2}{ }_{2} \mathrm{Zn}$ gegenüber $\mathrm{Cp}^{*}{ }_{2} \mathrm{Zn}_{2}$.

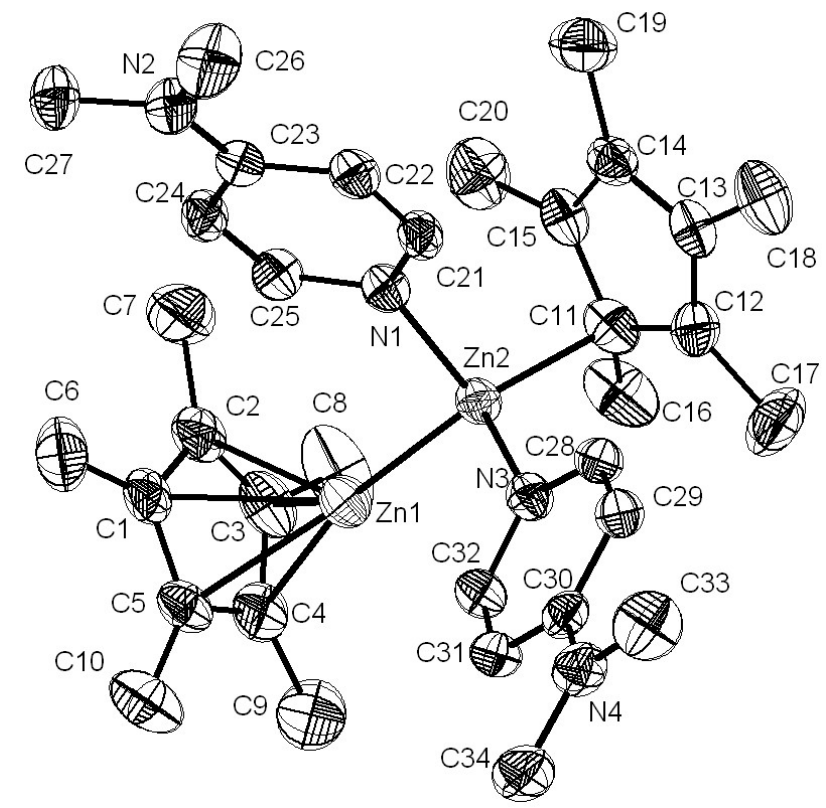

Abb. 1. Molekülstruktur von 2. H-Atome sind nicht gezeigt. Ausgewählte Bindungslängen $[\AA]$ und -winkel $\left[^{\circ}\right]$ : Zn1-Zn2 2.418(1), Zn1-C1 2.425(2), Zn1-C2 2.395(2), Zn1-C3 2.328(2), Zn1-C4 2.300(2), Zn1C5 2.360(2), Zn2-C11 2.174(2), Zn2-N1 2.115(2), Zn2-N3 2.125(2);

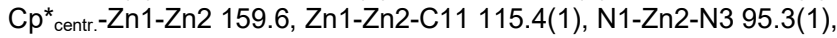
N1-Zn2-Zn1105.0(1), N3-Zn2-Zn1 110.1(1), N1-Zn2-C11 113.9(1), N3-Zn2-C11 115.0(1).

Einkristalle wurden aus Lösungen in $n$-Pentan/Toluol (2) bzw. $n$-Pentan (3) nach Lagerung bei $-30{ }^{\circ} \mathrm{C}$ erhalten. ${ }^{[15]}$ Das $\mathrm{Zn}$-Atom in 3 ist von einem dmap-Liganden sowie zwei $\eta^{2}$-gebundenen $\mathrm{Cp}^{*}$ Gruppen (Zn1-C10 2.090(5), Zn1-C12 2.483(5); Zn1-C20 2.169(5), Zn1-C22 2.300(5) Å) koordiniert. Im Gegensatz dazu bildet sich mit dem sterisch weniger anspruchsvollen $\mathrm{ZnMe}_{2}$ das Bisaddukt (dmap) ${ }_{2} \mathrm{ZnMe}_{2}{ }^{[16]}$ In 2 koordinieren dagegen überraschenderweise zwei Lewis-Basen (dmap) geminal an ein Zn-Atom (Zn2), während das zweite $\mathrm{Zn}$-Atom ( $\mathrm{Zn} 1)$ lediglich einen $\eta^{5}$-gebundenen $\mathrm{Cp}^{*}$ Liganden trägt ( $\mathrm{Cp}^{*}$ centr. $-Z n 12.033 \AA$; vergl. $2.04 \AA$ in 1). ${ }^{[17]} \mathrm{Das}$ Zn1-Atom liegt im Gegensatz zu 1 nicht zentriert über dem Cp*Liganden, so dass die Zn-C $\mathrm{Cp}$ *-Bindungslängen $(2.300(2)-2.425(2)$ $\AA)$ stärker variieren als die in $1(2.27-2.30 \AA) \cdot{ }^{[1 a]}$ Die Koordinationssphäre von $\mathrm{Zn} 2$ wird von einem $\sigma$-gebundenen $\mathrm{Cp}^{*}$-Rest (Zn2C11 2.174(2) A) komplettiert, die Abstände zu den nächsten RingC-Atomen (Zn2-C15 2.696(3), Zn2-C12 2.773(3) Å) sind deutlich länger. Die Zn-N-Bindungslängen in 2 (Zn2-N1 2.115(2), Zn2-N3

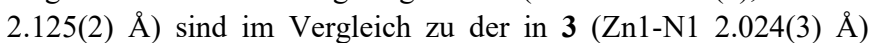
signifikant verlängert, jedoch kürzer als zum vierfach-koordinierten
Zn-Atom in $\mathrm{Me}_{2} \mathrm{Zn}(\mathrm{dmap})_{2}(\mathrm{Zn} 1-\mathrm{N} 1 / 22.177(2) \AA) .{ }^{[16]}$ Die zentrale $\mathrm{Zn1-Zn2}$ Bindungslänge ist infolge der Erhöhung der Koordinationszahl an Zn2 mit 2.418(1) $\AA$ im Vergleich zu der in 1 (2.305(3) $\AA$ ) um über $11 \mathrm{pm}$ aufgeweitet. Dieser Abstand ist länger als der aller bekannten $\mathrm{Zn}_{2}$-Verbindungen, die typischerweise $\mathrm{Zn}-\mathrm{Zn}$ Abstände von 2.29-2.35 $\AA$ aufweisen, ${ }^{[1-7]}$ allerdings vergleichbar zu $\mathrm{Zn}$-Zn-Abständen in H-verbrückten Organozinkhydriden $[\mathrm{RZnH}]_{2}$ $\left(\mathrm{R}=\left[2,6-\left(2,6-i-\mathrm{Pr}_{2} \mathrm{C}_{6} \mathrm{H}_{3}\right)-\mathrm{C}_{6} \mathrm{H}_{3}\right] \quad 2.408(1) \quad \AA_{,},{ }^{[3]} \quad[\{(2,6-i-\right.$ $\left.\left.\left.\mathrm{Pr}_{2} \mathrm{C}_{6} \mathrm{H}_{3}\right) \mathrm{N}(\mathrm{Me}) \mathrm{C}\right\}_{2} \mathrm{CH}\right] 2.451(1) \AA^{[18]}$ ). Im Gegensatz zur nahezu linearen Struktur von $1\left(177.4(1)^{\circ}\right)$ zeigt 2 infolge der Koordination der dmap Moleküle eine deutliche Abweichung von der linearen Anordnung. Hierbei ist die Abwinklung des $\eta^{5}$-gebundenen $\mathrm{Cp}^{*}$ Liganden ( $\left.\mathrm{Zn} 2-\mathrm{Zn} 1-\mathrm{Cp}^{*}{ }_{\text {centr. }} \quad 159.6^{\circ}\right)$ erwartungsgemäß weniger stark ausgeprägt als die des $\sigma$-gebundenen $\mathrm{Cp}^{*}$-Liganden $(\mathrm{Zn} 1-\mathrm{Zn} 2$ C11 115.4 $\left.(1)^{\circ}\right)$. Die dmap-Moleküle stehen nahezu orthogonal zueinander, wobei der N1-Zn2-N3 Bindungswinkel $\left(95.3(1)^{\circ}\right)$ signifikant kleiner ist als die N-Zn2-Zn1- (105.0(1), 110.1(1) $\left.{ }^{\circ}\right)$ und $\mathrm{N}$ Zn2-C11-Bindungswinkel (113.9(1), 115.0(1) $\left.{ }^{\circ}\right)$.

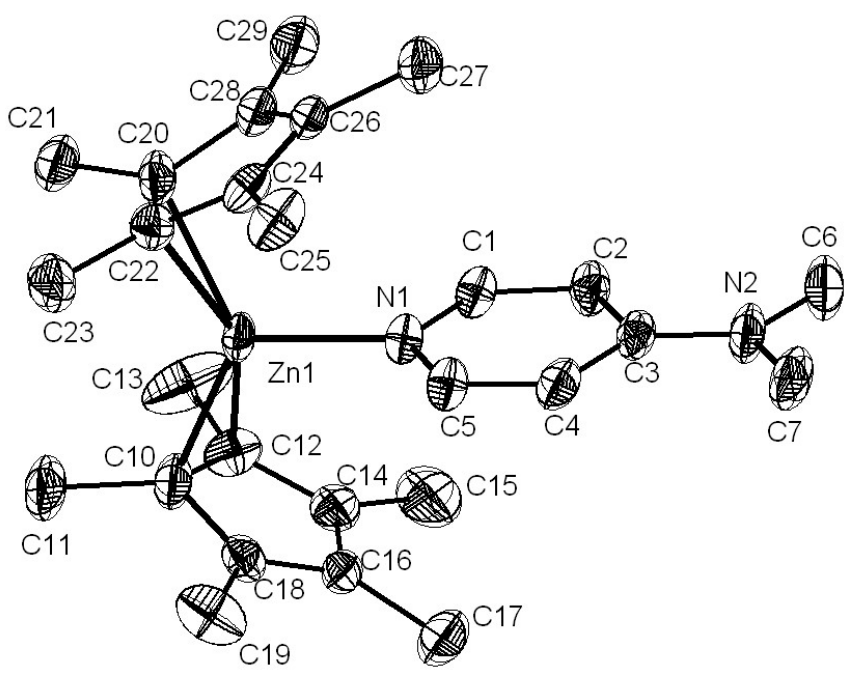

Abb. 1. Molekülstruktur von 3. H-Atome sind nicht gezeigt. Ausgewählte Bindungslängen $[\AA]$ und -winkel [ $\left.{ }^{\circ}\right]$ : Zn1-C10 2.090(5), Zn1C12 2.483(5), Zn1-C20 2.169(5), Zn1-C22 2.300(5), Zn1-N1 2.024(3); N1-Zn1-C10 115.6(2), N1-Zn1-C20 115.8(2).

Um die Bildung und die Bindungsverhältnisse von $\mathbf{2}$ besser zu verstehen, wurden DFT Rechnungen durchgeführt. ${ }^{[19]}$ Die Bildung von $\mathrm{Cp}_{2}{ }_{2} \mathrm{Zn}_{2}$ (dmap) 1' durch Koordination von einem dmapMolekül an $\mathrm{Cp}_{2}{ }_{2} \mathrm{Zn}_{2} 1$ verläuft exotherm $(-4.6 \mathrm{kcal} / \mathrm{mol})$. Die $\mathrm{Zn}-$ Zn-Bindungslänge bleibt hierbei nahezu konstant (von $2.36 \mathbf{1}$ auf $2.371^{\prime} \AA \AA$ ), allerdings kommt es zu einer Ladungsverschiebung. In 1 tragen beide $\mathrm{Zn}$-Atome eine Ladung von 0.89 , während in 1' das dmap-koordinierte Zn-Atom elektropositiver (0.99) ist als das zweite Zn-Atom (0.76). Die Koordination eines zweiten dmap-Moleküls in vicinaler Position verläuft endotherm $(+2.6 \mathrm{kcal} / \mathrm{mol})$, während für die geminale Anordnung eine stark exotherme Reaktion berechnet wird (-9.2 kcal/mol). Die Bildung von 2 mit geminaler Koordination der Lewis-Basen ist demzufolge mit $-13.8 \mathrm{kcal} / \mathrm{mol}(-57.7$ $\mathrm{KJ} / \mathrm{mol})$ signifikant günstiger als die vicinale Anordnung (-2.0 $\mathrm{kcal} / \mathrm{mol}$ ). Die $\mathrm{Zn}-\mathrm{Zn}$-Bindungslänge von 2 wird infolge der Koordination des zweiten dmap Moleküls auf $2.44 \AA$ aufgeweitet, was sehr gut mit dem experimentell bestimmten Abstand 2.418(1) $\AA$ übereinstimmt. Beide $\mathrm{Zn}$-Atome tragen eine stark unterschiedliche Ladung, wobei Zn1 (0.60) im Vergleich zum dmap-koordinierten 
Zn2 (1.15) erwartungsgemäß eine deutlich höhere Ladungsdichte aufweist. Die NBO-Analyse zeigt eine Abnahme der Bindungsordnung von $0.93(96.7 \% \mathrm{~s}, 2.1 \% \mathrm{p}, 1.2 \% \mathrm{~d})$ in $1 \mathrm{zu} 0.85(92.9 \% \mathrm{~s}$, $7.0 \% \mathrm{p}, 0.1 \% \mathrm{~d}$ ) in 2 aufgrund einer Besetzung des $\mathrm{Zn}-\mathrm{Zn}$ antibindenden LUMO's, was gut mit publizierten Bindungsanalysen von 1 übereinstimmt. ${ }^{[1 b]}$

Mit der überraschenden Bildung von 2 wurde erstmalig eine Reaktion von $\mathrm{Cp}_{2}{ }_{2} \mathrm{Zn}_{2} 1$ realisiert, die unter Erhalt der $\mathrm{Zn}-\mathrm{Zn}$-Bindung verläuft. Die stark exotherme Bildung von 2 legt nahe, zukünftig weitere Addukte mit starken Lewis-Basen synthetisieren zu können und detailliertere Kenntnisse über die Reaktivität von 1 und die chemischen Stabilität der Zn-Zn Bindung zu erhalten.

\section{Experimentelles}

Alle Arbeiten wurden unter Argon-Atmosphäre durchgeführt. Lösungsmittel wurden über $\mathrm{Na} / \mathrm{K}$-Legierung getrocknet und vor $\mathrm{Ge}-$ brauch entgast. ${ }^{1} \mathrm{H}$ und ${ }^{13} \mathrm{C}\left\{{ }^{1} \mathrm{H}\right\}$ NMR-Spektren wurden auf einem Bruker Avance 500 Spektrometer aufgenommen und gegen die Resonanzen des Lösungsmittels $\left(\mathrm{C}_{6} \mathrm{D}_{5} \mathrm{H}:{ }^{1} \mathrm{H}: \delta=7.154 ;{ }^{13} \mathrm{C}: \delta=\right.$ 128.0) referenziert. IR-Spektren wurden auf einem ALPHA-T FT-IRSpektrometer. Schmelzpunkte wurden in versiegelten Kapillaren bestimmt und sind nicht korrigiert.

2: Eine Lösung von $0.20 \mathrm{~g} \mathrm{Cp}_{2}{ }_{2} \mathrm{Zn}_{2}(0.5 \mathrm{mmol})$ in $5 \mathrm{~mL}$ Pentan wird bei $0{ }^{\circ} \mathrm{C}$ mit einer Lösung von $0.24 \mathrm{~g} \mathrm{dmap}(2.0 \mathrm{mmol})$ in $5 \mathrm{~mL}$ Toluol versetzt, $2 \mathrm{~h}$ bei $-25^{\circ} \mathrm{C}$ gerührt, mit weiteren $5 \mathrm{~mL}$ Pentan versetzt und anschließend bei $-30{ }^{\circ} \mathrm{C}$ gelagert. Hellgelbe Kristalle von 2.Toluol werden nach 7 Tagen erhalten.

Ausbeute (isolierte Kristalle) $0.08 \mathrm{~g}(0.12 \mathrm{mmol}, 25 \%)$. Schmelzpunkt: $105{ }^{\circ} \mathrm{C} .{ }^{1} \mathrm{H}-\mathrm{NMR}\left(500 \mathrm{MHz}, \mathrm{C}_{6} \mathrm{D}_{6}, 25^{\circ} \mathrm{C}\right): \delta=2.03\left(\mathrm{~s}, 30 \mathrm{H}, \mathrm{C}_{5} \mathrm{Me}_{5}\right)$, 2.09 (s, 1.5H, Toluol), $2.20(\mathrm{~s}, 12 \mathrm{H}, \mathrm{NMe}), 6.09$ (dd, ${ }^{3} \mathrm{~J}_{\mathrm{HH}}=6.3 \mathrm{~Hz}$, $\left.{ }^{3} \mathrm{~J}_{\mathrm{HH}}=1.6 \mathrm{~Hz}, 4 \mathrm{H}, \mathrm{C}(3)-\mathrm{H}\right), 6.89-7.20\left(\mathrm{~m}, 2.5 \mathrm{H}\right.$, Toluol), 8.46 (dd, ${ }^{3} \mathrm{~J}_{\mathrm{HH}}$ $\left.=6.3 \mathrm{~Hz},{ }^{3} \mathrm{~J}_{\mathrm{HH}}=1.6 \mathrm{~Hz}, 2 \mathrm{H}, \mathrm{C}(2)-\mathrm{H}\right) .{ }^{13} \mathrm{C}\left\{{ }^{1} \mathrm{H}\right\}-\mathrm{NMR}\left(125 \mathrm{MHz}, \mathrm{C}_{6} \mathrm{D}_{6}\right.$ $\left.25^{\circ} \mathrm{C}\right): \delta=10.4\left(\mathrm{C}_{5} \mathrm{Me}_{5}\right), 38.3\left(\mathrm{NMe}_{2}\right), 106.8(\mathrm{C}(3)), 109.0\left(\mathrm{C}_{5} \mathrm{Me}_{5}\right)$, $150.5(\mathrm{C}(2)), 154.3(\mathrm{C}(4))$. IR (Nujol): $v=2853,1611,1461,1377$ $1224,1013,802 \mathrm{~cm}^{-1}$.

3: Eine Lösung von $0.67 \mathrm{~g} \mathrm{Cp}^{*} \mathrm{Zn}(2.0 \mathrm{mmol})$ und $0.48 \mathrm{~g}$ dmap $(4.0$ $\mathrm{mmol}$ ) in $15 \mathrm{~mL}$ Hexan werden unter Rückfluß erhitzt und anschließend bei $-30^{\circ} \mathrm{C}$ gelagert. Farblose Kristalle von 2 werden nach $36 \mathrm{~h}$ erhalten.

Ausbeute (isolierte Kristalle) $0.49 \mathrm{~g}(1.1 \mathrm{mmol}, 55 \%)$. Schmelzpunkt: $205{ }^{\circ} \mathrm{C}$. ${ }^{1} \mathrm{H}-\mathrm{NMR}\left(500 \mathrm{MHz}, \mathrm{C}_{6} \mathrm{D}_{6}, 25^{\circ} \mathrm{C}\right): \delta=1.99\left(\mathrm{~s}, 6 \mathrm{H}, \mathrm{NMe}_{2}\right)$, $2.08\left(\mathrm{~s}, 30 \mathrm{H}, \mathrm{C}_{5} \mathrm{Me}_{5}\right), 5.68\left(\mathrm{dd},{ }^{3} \mathrm{~J}_{\mathrm{HH}}=6.9 \mathrm{~Hz},{ }^{3} \mathrm{~J}_{\mathrm{HH}}=1.6 \mathrm{~Hz}, 2 \mathrm{H}\right.$, $\mathrm{C}(3)-\mathrm{H}), 7.22\left(\mathrm{dd},{ }^{3} \mathrm{~J}_{\mathrm{HH}}=6.9 \mathrm{~Hz},{ }^{3} \mathrm{~J}_{\mathrm{HH}}=1.6 \mathrm{~Hz}, 2 \mathrm{H}, \mathrm{C}(2)-\mathrm{H}\right) .{ }^{13} \mathrm{C}\left\{{ }^{1} \mathrm{H}\right\}$ NMR (125 MHz, $\left.\mathrm{C}_{6} \mathrm{D}_{6}, 25^{\circ} \mathrm{C}\right): \delta=12.3\left(\mathrm{C}_{5} \mathrm{Me}_{5}\right), 38.3\left(\mathrm{NMe}_{2}\right), 105.3$ (C(3)), $114.1\left(C_{5} \mathrm{Me}_{5}\right), 147.5(\mathrm{C}(2)), 154.5(\mathrm{C}(4)) . \mathrm{IR}$ (Nujol): $v=2923$, $1623,1546,1440,1390,1261,1228,1075,1021,801,604 \mathrm{~cm}^{-1}$.

Received: ((will be filled in by the editorial staff))

Published online on ((will be filled in by the editorial staff))

Keywords: Niedervalente Verbindungen - Lewis Säuren · Zink · Röntgenbeugung

[1] a) I. Resa, E. Carmona, E. Gutierrez-Puebla, A. Monge, Science 2004, 305, 1136-1138; b) D. del Río, A. Galindo, I. Resa, E. Carmona, $A n-$ gew. Chem. 2005, 117, 1270-1273; Angew. Chem. Int. Ed. 2005, 44, 1244-1247. Siehe auch folgenden Übersichtsartikel: E. Carmona, A. Galindo, Angew. Chem. 2008, 120, 6626-6637; Angew. Chem. Int. Ed. 2008, 47, 6526-6536.

[2] A. Grirrane, I. Resa, A. Rodriguez, E. Carmona, E. Alvarez, E. Gutierrez-Puebla, A. Monge, A. Galindo, D. del Río, R. A. Andersen, J. Am. Chem. Soc. 2007, 129, 693-703.

[3] a) Z. Zhu, R. J. Wright, M. M. Olmstead, E. Rivard, M. Brynda, P. P. Power, Angew. Chem. 2006, 118, 5939-5942; Angew. Chem. Int. Ed. 2006, 45, 5807-5810; b) Z. Zhu, M. Brynda, R. J. Wright, R. C. Fisch- er, W. A. Merrill, E. Rivard, R. Wolf, J. C. Fettinger, M. M. Olmstead, P. P. Power, J. Am. Chem. Soc. 2007, 129, 10847-10857.

[4] Y. Wang, B. Quillian, P. Wei, H. Wang, X.-J. Yang, Y. Xie, R. B. King, P. v. R. Schleyer, H. F. Schaefer, III, G. H. Robinson, J. Am. Chem. Soc. 2005, 127, 11944-11945.

[5] X.-J. Yang, J. Yu, Y. Liu, Y. Xie, H. F. Schaefer, Y. Liang, B. Wu, Chem. Commun. 2007, 2363-2365.

[6] Y.-C. Tsai, D.-Y. Lu, Y.-M. Lin, j.-K. Hwang, J.-S. K. Yu, Chem. Commun. 2007, 4125-4127.

[7] I. L. Fedushkin, A. A. Skatova, S. Y. Ketkov, O. V. Eremenko, A. V. Piskunov, G. K. Fukin, Angew. Chem. 2007, 119, 4380-4383; Angew. Chem. Int. Ed. 2007, 46, 4302-4305.

[8] a) Y. Kan, J. Mol. Struct. Theochem 2007, 805, 127-132; b) A. Velazquez, I. Fernández, G. Frenking, G. Merino, Organometallics 2007, 26, 4731-4736; c) M. R. Philpott, Y. Kawazoe, Chem. Phys. 2007, 333 , 201-207; d) K. N. Pandey, J. Organomet. Chem. 2007, 692, 10581063; e) H. Wang, C. Yang, B. Wan, K.-L- Han, J. Theor. Comp. Chem. 2006, 5, 461-473; f) M. R. Philpott, Y. Kawazoe, J. Mol. Struct. Theochem 2006, 776, 113-123; g) J. W. Kress, J. Phys. Chem. A 2005, $109,7757-7763 ;$ h) Y. Xie, H. F. Schaefer, III, R. B. King, J. Am. Chem. Soc. 2005, 127, 2818-2819.

[9] a) T. Eisenmann, J. Khanderi, S. Schulz, U. Flörke, Z. Anorg. Allg. Chem. 2008, 634, 507-513; b) M. Münch, U. Flörke, M. Bolte, S. Schulz, D. Gudat, Angew. Chem. 2008, 120, 1535-1539; Angew. Chem. Int. Ed. 2008, 47, 1512-1516; c) S. Schulz, M. Münch, U. Flörke, Z. Anorg. Allg. Chem., im Druck.

[10] a) P. Jutzi, N. Burford, Chem. Rev. 1999, 99, 969-990; b) P. H. M. Budzelaar, J. J. Engelberts, J. H. van Lenthe, Organometallics 2003, 22, 1562-1576.

[11] a) F. Thomas, S. Schulz, M. Nieger, Eur. J. Inorg. Chem. 2001, 161166; b) S. Schulz, M. Nieger, Organometallics 2000, 19, 2640-2643.

[12] a) M. Krieger, R. O. Gould, B. Neumüller, K. Harms, K. Dehnicke, Z. Anorg. Allg. Chem. 1998, 624, 1434-1442; b) A. Looney, R. Han, I. B. Gorrell, M. Cornebise, K. Yoon, G. Parkin, A. L. Rheingold, Organometallics 1995, 14, 274-288; c) R. Han, I. B. Gorrell, A. Looney, G. Parkin, Chem. Commun. 1991, 717-719; d) W. Kläui, U. Schilde, M. Schmidt, Inorg. Chem. 1997, 36, 1598-1601.

[13] Temperaturabhängige NMR-Spektren von 2 finden sich im elektronischen Supplement.

[14] a) M. B. Power, J. R. Nash, M. D. Healy, A. R. Barron, Organometallics 1992, 11, 1830; b) A. Kuczkowski, S. Schulz, Martin Nieger, P. R. Schreiner, Organometallics 2002, 21, 1408. Die Daten sind im Supplement zusammengefasst.

[15] Bruker AXS SMART APEX CCD Diffraktometer $\left(\mathrm{Mo}_{\mathrm{K \alpha}}\right.$-Strahlung, $\lambda$ $=0.71073 \AA ; T=203(2)$ 2, 153(2) K 3). Strukturen wurden mittels Direkter Methoden gelöst (SHELXS-97, G. M. Sheldrick, Acta Crystallogr. Sect. A 1990, 46, 467) und mit allen unabhängigen Strukturfaktoren $\left(\mathrm{F}^{2}\right)$ verfeinert. Semiempirische Absorptionskorrekturen wurden durchgeführt; alle Nichtwasserstoffatome wurden anisotrop verfeinert und die Wasserstoffatome mittels eines Reitermodells (SHELXL-97, Program for Crystal Structure Refinement, G. M. Sheldrick, Universität Göttingen, 1997). 2: $\mathrm{C}_{34} \mathrm{H}_{50} \mathrm{~N}_{4} \mathrm{Zn}_{2} \cdot 0.25 \mathrm{C}_{7} \mathrm{H}_{8}, M$ $=666.54$, farblose Kristalle $(0.28 \times 0.22 \times 0.13 \mathrm{~mm})$; triklin, Raumgruppe $P-1 ; a=10.8055(6), b=11.1214(6), c=17.1985(9) \AA ; \alpha=$ 71.822(2), $\beta=89.356(3), \gamma=75.260(3)^{\circ}, V=1893.78(18) \AA^{3} ; Z=2$; $\mu=1.293 \mathrm{~mm}^{-1} ; \rho_{\text {ber. }}=1.169 \mathrm{~g} \mathrm{~cm}^{-3} ; 73213$ Reflexe $\left(2 \theta_{\max }=58^{\circ}\right)$, 11020 unabhängig $\left(R_{\text {int }}=0.0640\right) ; 395$ Parameter; $\max . / \mathrm{min}$. Restelektronendichte $1.054 \mathrm{e}^{-3} /-0.285 \mathrm{e}^{-3} ;$ max./min. Transmission $0.8816 / 0.7820 ; R_{1}=0.0463(I>2 \sigma(I)), w R_{2}($ alle Daten $)=0.1367$. Fehlgeordnetes Toluol (C61-C64) mit Inversionszentrum; SOF 0.5. 3: $\mathrm{C}_{27} \mathrm{H}_{40} \mathrm{~N}_{2} \mathrm{Zn}, M=457.98$, farblose Kristalle $(0.48 \times 0.44 \times 0.13 \mathrm{~mm})$; Triklin, Raumgruppe $P-1 ; a=9.848(5), b=10.104(6), c=14.489$ (8) $\AA ; \alpha=98.791(11), \beta=98.449(11), \gamma=111.355(11)^{\circ}, V=1294.4(12)$ $\AA^{3} ; Z=2 ; \mu=0.963 \mathrm{~mm}^{-1} ; \rho_{\text {ber }}=1.175 \mathrm{~g} \mathrm{~cm}^{-3} ; 10044$ Reflexe $\left(2 \theta_{\max }\right.$ $\left.=59^{\circ}\right), 5669$ unabhängig $\left(R_{\text {int }}=0.0826\right) ; 283$ Parameter; max. $/$ min. Restelektronendichte $0.989 \mathrm{e} \AA^{-3} /-0.560 \mathrm{e}^{-3} ;$ max./min. Transmission $0.8850 / 0.6549 ; R_{1}=0.0253(I>2 \sigma(I)), w R_{2}$ (alle Daten $)=0.0695$. Die kristallographischen Daten (ohne Strukturfaktoren) der in dieser Veröffentlichung beschriebenen Strukturen wurden als ,supplementary publication no. CCDC-694349 (2) und CCDC-694247 (3)“ beim Cambridge Crystallographic Data Centre hinterlegt. Kopien der Daten können kostenlos bei folgender Adresse in Großbritannien angefordert 
werden: CCDC, 12 Union Road, Cambridge, CB21EZ (Fax: (+44)1223/336033; E-mail: deposit@ccdc.cam-ak.uk).

[16] F. Thomas, S. Schulz, M. Nieger, Angew. Chem. 2005, 117, 58135815; Angew. Chem. Int. Ed. 2005, 44, 5668-5670.

[17] Parallel zu dieser Arbeit berichten Jones et al. über Reaktionen einer niedervalenten $\mathrm{Mg}$-Verbindung mit vier verschiedenen Lewis-Basen bei denen ausnahmslos die erwarteten vicinalen Bisaddukte gebildet wurden. Diese wurden durch Einkristallröntgenstrukturanalyse zweifelsfrei charakterisiert. Angew. Chem. 2008, im Druck, DOI: 10.1002 /ange. 200803960 .
[18] H. Hao, C. Cui, H. W. Roesky, G. Bai, H.-G. Schmidt, M. Noltemeyer, Chem. Commun. 2007, 1118-1119.

[19] DFT-Rechungen wurden mit dem Gaussian03 Programmpaket (M.J. Frisch, et al. Gaussian 03, Revision D.02; Gaussian Inc.: Pittsburgh, PA, 2003, vollst. Zitat im Supplement) durchgeführt. Molekülstrukturen wurden auf B3LYP/SVP-Niveau energieoptimiert; SVP ist der split-valence Basissatz mit zusätzlichen Polarisationsfunktionen von Ahlrichs et al. Berechnete Ladungsdichten aus NBO Populationsanalysen für 2 werden im Supplement angegeben. 
Niedervalente Lewis-Säure

Daniella Schuchmann, Ulrich Westphal, Stephan Schulz, ${ }^{*}$ Ulrich Flörke, Dieter Bläser und Roland Boese

Page - Page

Erste Reaktion von Dizinkocen unter Erhalt der Zn-Zn-Bindung

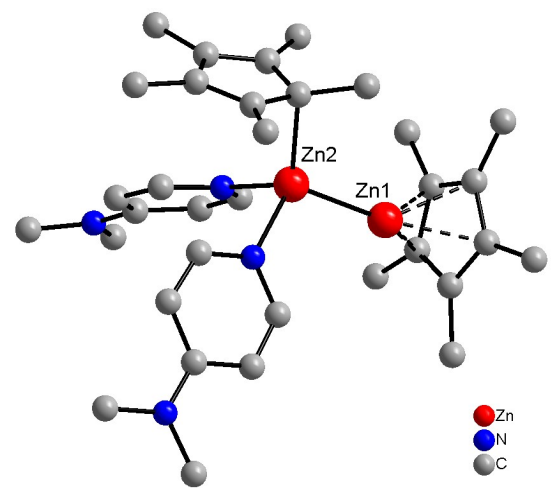

$C{ }^{*}{ }_{2} Z n_{2} 1$ reagiert mit einem vierfachen Überschuss der starken Lewis-Base 4Dimethylaminopyridin (dmap) unter Bildung des unerwarteten Lewis SäureBase Adduktes 2, in dem zwei dmapMoleküle unter Erhalt der Zn-ZnBindung geminal an ein $\mathrm{Zn}$-Zentrum binden. 


\section{DuEPublico}

Duisburg-Essen Publications online

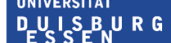

offen im Denken

\section{$\mathbf{U b}$ universitäts}

Dieser Text wird über DuEPublico, dem Dokumenten- und Publikationsserver der Universität Duisburg-Essen, zur Verfügung gestellt. Die hier veröffentlichte Version der EPublikation kann von einer eventuell ebenfalls veröffentlichten Verlagsversion abweichen.

DOI: $\quad 10.1002$ /ange.200803736

URN: urn:nbn:de:hbz:464-20201030-091520-3

This is the peer reviewed version of the following article: Angew. Chem. 2009,121, 821-824 which has been published in final form at: https://doi.org/10.1002/ange.200803736

Alle Rechte vorbehalten. 